\title{
Analysis of maintenance indicators in a public transport company in the city of Manaus
}

\author{
Edry Antonio García Cisneros ${ }^{1}$, Tirso Lorenzo Reyes Carvajal ${ }^{2}$, João Evangelista Netto ${ }^{3}$, Alain Ricote Paumier ${ }^{4}$, Ricardo \\ Wilson da Costa \\ Dr. ${ }^{1,3,5}$ Departamento de Ingeniería Mecánica, Escuela Superior de Tecnología, Universidad del Estado de Amazonas, Manaos \\ (brasil.edry1961cu@gmail.com, joao_evangelista_neto@yahoo.com,rwcruz@gmail.com) \\ Dr. ${ }^{2}$ Instituto de Tecnologia e Educação Galileo da Amazônia (ITEGAM). Avenida Joaquim Nabuco, No. 1950. CEP: 69005-080. \\ Manaus. Amazonas ( tirsolrca@gmail.com) \\ Dr. ${ }^{4}$ Departamento de Ingeniería Mecánica, Facultad de Electromecánica, Universidad de Camagüey, Cuba.
} (alain.ricote@reduc.edu.cu)

\begin{abstract}
Maintenance activity occupies a privileged place in the development of any carrier in the world and is considered as a key sector for its direct impact on obtaining productive results. Under current conditions, the need to reduce operating costs and increase productivity of transportation to achieve returns in line with current requirements is evident. However, there always has the right to provide maintenance management companies information quantitatively and qualitatively adequate to measure and indicate which of the maintenance actions are accomplished and what actions should be taken to improve such operations Tool that the maintenance work is achieved effectively. In this paper an experience developed in a company of public transport in the city of Manaus where applied indicators that tend just are provide information to management in the maintenance area for timely decision making and the results of the calculations are shown exposed some of the indicators. The analysis carried out shows the strategic importance of the use of these indices to ensure efficient and effective maintenance that allows reducing maintenance costs is in turn increase productivity.
\end{abstract}

Keywords: weldability; quality tools; SMT process; tablet.

\section{Análisis de indicadores de mantenimiento en una empresa de transporte colectivo en la ciudad de Manaos}

\section{RESUMEN}

La actividad de mantenimiento ocupa un lugar privilegiado en el desarrollo de cualquier empresa de transporte en el mundo y es considerado como un sector clave por su incidencia directa en la obtención de resultados productivos. En las condiciones actuales, resulta evidente la necesidad de reducir los costos operativos e incrementar la productividad de los medios de transporte para alcanzar rendimientos acordes a las exigencias actuales. Sin embargo, no siempre se cuenta con las herramientas adecuadas para proveer a la administración del mantenimiento de las empresas información cuantitativa y cualitativa adecuada que permita medir e indicar cuáles de las acciones del mantenimiento son logradas y que acciones se deben tomar para mejorar dichas operaciones para que se logre el trabajo de mantenimiento de forma eficaz. En este trabajo se expone una experiencia desarrollada en una empresa de transporte colectivo en la ciudad de Manaos donde son aplicados indicadores que tienden justamente dotar de información a la administración del área de mantenimiento para la oportuna toma de decisiones y se muestran los resultados de los cálculos de algunos de los indicadores. El análisis desarrollado muestra la importancia estratégica del uso de estos índices para asegurar un mantenimiento eficiente y eficaz que permita la reducción de los costes de mantenimiento es a su vez incrementar la productividad a la vez ratifica la necesidad de una adecuada gestión del mantenimiento.

Palabras clave: maintenance; transport, maintenance indicator.

\section{INTRODUCCIÓN}

\section{I.1 ACTIVIDAD DEL MANTENIMIENTO}

La actividad de mantenimiento es considerada un sector estratégico en las empresas de transporte. Manaos, es una ciudad del norte de Brasil donde la población asciende a más de dos millones y el medio de transporte colectivo utilizado sigue siendo el de ómnibus simples y articulados y por tanto este sector requiere de servicios eficientes para mantener los tiempos de espera de los pasajeros en niveles acordes a la media mundial para

ITEGAM - JETIA Vol. 02, Nº6. Junho de 2016. Manaus - Amazonas, Brasil. ISSN 2447-0228 (ONLINE). 
Edry Antonio García Cisneros et al/ ITEGAM-JETIA Vol.02, No 06, pp.55-60. Junho, 2016.

asegurar la calidad y eficiencia que los ciudadanos de la ciudad merecen. En esta ciudad existen varias empresas dedicadas al transporte colectivo y sus características difieren de acuerdo al tamaño y posibilidades económicas.

Los gobiernos locales y nacionales conscientes de esta situación aportan recursos para el desarrollo de investigaciones tendientes a mejorar la actividad del transporte y dentro de esta al tema del mantenimiento.

La sociedad brasilera de normas técnicas [1] define al mantenimiento como el conjunto de acciones necesarias para que un ítem sea conservado o restaurado de forma tal que pueda mantener su estado técnico específico.

En otras palabras, la actividad de mantenimiento debe siempre tender a mantener el buen estado técnico de un ítem determinado ya sea mediante el cambio o la restauración de este a parte de este para así garantizar el funcionamiento del equipo dentro de los parámetros técnicos para los cuales fue construido. En la práctica esto no siempre es posible y también no siempre se conocen las causas de estos problemas. Existen indicadores de mantenimiento que justamente cuantifican este proceso de mantenimiento, pero para obtenerlos es necesario contar con la documentación necesaria.

Las actividades de mantenimiento tienen la finalidad de mantener el equipo en condiciones adecuadas de funcionamiento. Estas actividades se pueden clasificadas como: Mantenimiento correctivo planificado y no planificado, mantenimiento preventivo, mantenimiento predictivo, mantenimiento detectivo y ingeniería de mantenimiento [7]. Estos autores definen el objetivo del mantenimiento como: el objetivo de garantizar a disponibilidad de funcionamiento de los equipos e instalaciones de forma tal que aseguren el proceso de producción o servicio con confiabilidad, seguridad preservando el medio ambiente y con costos adecuados.

Indicador es un instrumento que permite medir las modificaciones en las características de un sistema, o sea, los indicadores deben establecer, para un período determinado, una medida de la sustentabilidad del sistema [2] [3].

Ya [6], define los indicadores como los datos numéricos establecidos sobre algunos procesos que se deseen controlar.

Por su parte otros autores [4] [5] define los indicadores como guías que permiten medir la eficiencia de las acciones tomadas, así como medir la desviación entre lo programado y lo realizado.

Mediante los indicadores es posible entonces hacer comparaciones a lo largo del tiempo.

El propio [5] plantea que los indicadores utilizados en el mantenimiento desempeñan un papel importante para la evaluación de las actividades desarrolladas por esta función aportando información a la administración en el sentido de intensionar las acciones dirigidas a los cambios necesarios para el aumento de la eficiencia y la maximización de los resultados.
De acuerdo con [8] la implementación de indicadores usados por el sector de mantenimiento debe relacionarse con aquellos aspectos que la propia empresa entienda que son importantes e agreguen un valor a la gestión de este sector. No debe gastarse recursos en el levantamiento e implementación de indicadores que no contribuyan al desarrollo de las actividades de mantenimiento.

Es posible afirmar que existe concordancia entre los investigadores en cuanto a la necesidad e importancia de implementar indicadores en la actividad de mantenimiento que permitan de manera práctica y eficiente el desarrollo de acciones de mantenimiento tendientes a asegurar eficiencia, productividad e racionalidad en el uso de recursos.

Debido a la existencia de múltiples indicadores para evaluar la actividad de mantenimiento, es una práctica común el uso de algunos de ellos, entre estos los denominados de clase mundial por ser los más utilizadas por las empresas son: tiempo medio entre fallas, tiempo medio para reparación, tiempo medio para falla, indicador de disponibilidad, costo de mantenimiento por el valor de reposición y otros relacionados a la gestión de costos y de mano de obra.

En este trabajo se exponen los resultados del análisis desarrollado en una empresa de transporte colectivo de la ciudad de Manaos y se muestra la importancia estratégica del uso de estos índices para asegurar un mantenimiento eficiente y eficaz que permita la reducción de los costos de mantenimiento es a su vez incrementar la productividad, a la vez, se ratifica la necesidad de una adecuada gestión del mantenimiento.

\section{METODOLOGÍA}

La metodología desarrollada en el trabajo para el análisis del mantenimiento en la empresa se basó en la información disponible por la empresa. Se utilizó el método de análisis y síntesis de la información disponible, así como la estadística descriptiva. Los indicadores calculados muestran la real situación de la misma y posibilitan la toma de decisiones para las acciones de mejoras.

\section{II.1 CARACTERIZACIÓN GENERAL DE LA EMPRESA}

Esta entidad fue fundada en 1958, y actúa en Manaos a partir del año 2011, opera en 21 rutas en las zonas norte y nordeste de la ciudad y cuenta con una frota de 134 vehículos transportando aproximadamente 2300000 pasajeros anualmente. La frota actual posee una edad media de 10 años de explotación del total de medios técnicos 116 son de la marca Volkswagen 17.230, 32 vehículos Mercedes Ben 1722 y 10 Volkswagen micro 9.150. Todo el proceso de mantenimiento se desarrolla dentro de la propia empresa que posee los equipamientos e instalaciones adecuadas para tales fines. 
Edry Antonio García Cisneros et al/ ITEGAM-JETIA Vol.02, No 06, pp.55-60. Junho, 2016.

\section{II.2 DETERMINACIÓN DE LA SITUACIÓN PROBLÉMICA, CAMPO, OBJETO DE ESTUDIO Y OBJETIVO DE LA INVESTIGACIÓN}

Situación problémica:

El departamento de mantenimiento de la empresa donde se realizó la investigación que opera una frota de vehículos destinados para la transportación colectiva de pasajeros en la ciudad de manaos del Estado de Amazonas, Brasil, no adopta un plan de mantenimiento preventivo como es recomendado por los fabricantes del parque de vehículos y crea su plan basado fundamentalmente a partir de una perspectiva basada en la intervención con el objetivo de realizar reparaciones y realizadas frecuentemente una vez ocurridas las fallas en estos vehículos lo que trae como consecuencia que estas acciones sean del tipo de respuesta correctiva aunque sea mejor acciones preventivas como es sugerido por los fabricantes de estos vehículos. Además, se pudo observar que no siempre se cuenta con la información necesaria que posibilite la adopción de otras acciones preventivas.

En la empresa no existe un banco de datos para las acciones de mantenimiento que posibilite profundizar en informaciones relacionadas con los intervalos de sustitución de todos los ítems de estos vehículos para las diferentes situaciones de operación de estos medios, además la verificación de las condiciones técnicas de estos son realizadas de forma empírica cuando los operadores o choferes inician el proceso de trabajo de estos vehículos es por ello que prima la condición correctiva que es realizada una vez detectada la falla. Los ítems donde se constató mayores frecuencias de ocurrencias de fallas fueron el motor, la caja de velocidades y el puente propulsor respectivamente.

Derivada de esta problemática se determina como campo de la investigación el proceso de mantenimiento de vehículos de la empresa y como objeto de estudio el ítem motor, el ítem caja y el ítem puente propulsor.

Como objeto de la investigación se plantea entonces la evaluación del proceso de mantenimiento del objeto de estudio mediante el uso de indicadores de mantenimiento.

\section{II.3 SELECCIÓN DE LOS INDICADORES DE MANTENIMIENTO DE LA EMPRESA}

Para cumplimentar el objetivo propuesto se utilizaron los siguientes indicadores:

- Frecuencia de fallas en el ítem motor, en el ítem caja de velocidades y en el ítem puente propulsor;

- Tiempo medio entre fallas;(TMEF)

- Tiempo medio para reparación;(TMR)

-Disponibilidad Física u operacional

\section{II.4 COLECCIÓN DE DATOS}

Para el desarrollo de la investigación se realizó la colecta de datos en el período comprendido entre los meses de enero y octubre del año 2014. Fueron consultados los documentos disponibles por la empresa contentiva de informaciones esenciales para el estudio de la política de mantenimiento adoptada y se tomó como referencia la información suministrada sobre el historial de mantenimiento de los ómnibus, los tiempos de paradas para la reparación y los costos de estas operaciones. Además, se recopiló información sobre las fallas y los servicios de mantenimientos de los ítems seleccionados cono objeto de estudio (motor, caja y puente propulsor) en el período observado. La investigación se centro en el estudio de estos ítems en los ómnibus modelo VW 17.230 por representar el 73,41\% del parque de vehículos existentes en la empresa.

\section{II.5 PROCESAMIENTO DE LA INFORMACIÓN}

Para el procesamiento de la información fue utilizada la Macro Solver y el sistema Microsoft Excel por las ventajas del mismo como disponibilidad, posibilidades en el tratamiento estadístico de la información, y rapidez en el procesamiento de la información y las posibilidades de salidas graficas e numéricas de los resultados.

\section{RESULTADOS}

En posición de los datos colectados en los registros de mantenimiento en el período de enero a octubre de 2014 fue posible realizar el levantamiento de los indicadores de ocurrencias de fallas en este período para los ítems motor, caja de velocidades y puente propulsor (Gráfico 1).

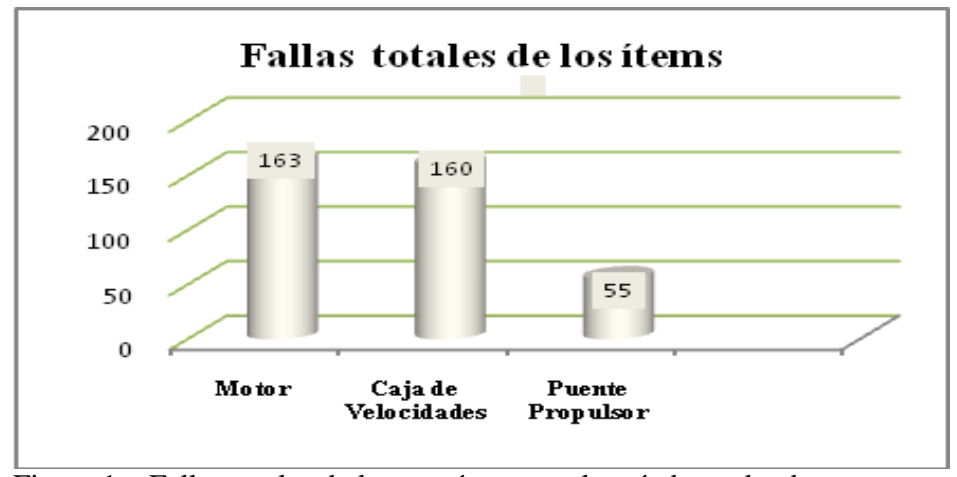

Figura 1 - Fallas totales de los tres ítems en el período evaluado. Fuente: Autores, (2016).

La gráfica muestra que las mayores ocurrencias de fallas en el período corresponden a los ítems motor y caja de velocidades con 163 y 160 fallas respectivamente mientras que la menor fue en el ítem puente propulsor con 55 fallas.

Los principales problemas relacionados con las fallas del ítem motor diesel MWM según el levantamiento de datos realizado están relacionadas con bajo niveles de aceite en el motor por fugas en las tuberías, fallas en las bombas de aceite, obstrucciones en los orificios de captura del aceite en el Carter y pérdida de presión en el bombeo a los cojinetes por excesivas holguras en estos y también en las bielas. Estas fallas sugieren falta de sistematicidad en las labores de mantenimiento. 
En la caja de velocidades las principales fallas están asociadas a desgastes en los engranes de las marchas centradas fundamentalmente en la tercera y cuarta marcha.

En el estudio del puente propulsor las principales fallas están asociadas también a desgastes tanto en la corona del diferencial como en el piñón de ataque del mismo.

Las probables causas de estas fallas pueden relacionarse a problemas de operación de los ómnibus y al proceso de envejecimiento de esta técnica.

Estos ómnibus son utilizados en la ciudad donde las condiciones viales no son óptimas, la calidad técnica de las vías no son las mejores en la ciudad por lo que es posible apreciar la existencia de insuficiencias como estrechez de las calles, irregularidades y obstrucciones en las vías de transportación dados por el envejecimiento de la ciudad y la falta de mantenimiento de la red vial.

También es posible relacionar el sobre uso de los ómnibus en los llamados horarios picos de la ciudad donde ocurre el mayor tránsito (entre las 7.00 am y $9.00 \mathrm{am}$; las $11.00 \mathrm{am}$ y las 15.00 horas y entre las 17.00 y 20.00 horas) y que corresponden también a los períodos de mayor aglomeración de la población y por consiguiente de mayor nivel de ocupación de las capacidades de carga de estos vehículos que en ocasiones llegan a sobre uso de estas capacidades.

Además de estos factores se puede plantear también las insuficiencias en la sistematicidad y selección adecuada del tipo de actividad de mantenimiento en la empresa, así como el envejecimiento de los ómnibus.

Tomando como base cada uno de los ítems individualmente, fueron levantadas las Frecuencias de fallas por ítems (Figuras $2,3$ e 4$)$ :

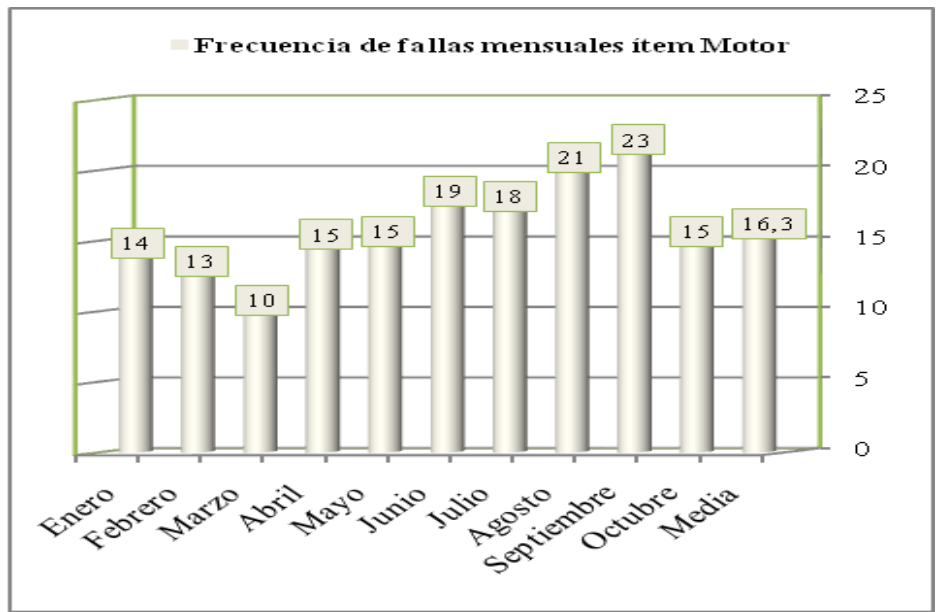

Figura 2 - Frecuencia de fallas en los meses evaluados. Ítem Motor. Fuente: Autores, (2016).

Como evidencian los resultados de las graficas 2,3 e 4 la frecuencia de fallas en el ítem resultó más significativo en los meses de agosto y setiembre oscilando entre 19 y 23 el número de frecuencia de fallas con una media mensual de 16.3 fallas que demandaron intervenciones correctivas, por su parte en el ítem caja de velocidades igualmente resultó significativo el incremento de la frecuencia de fallas en los meses de junio a agosto oscilando entre 23 y 20 la frecuencia y el valor medio se fijó en 16.

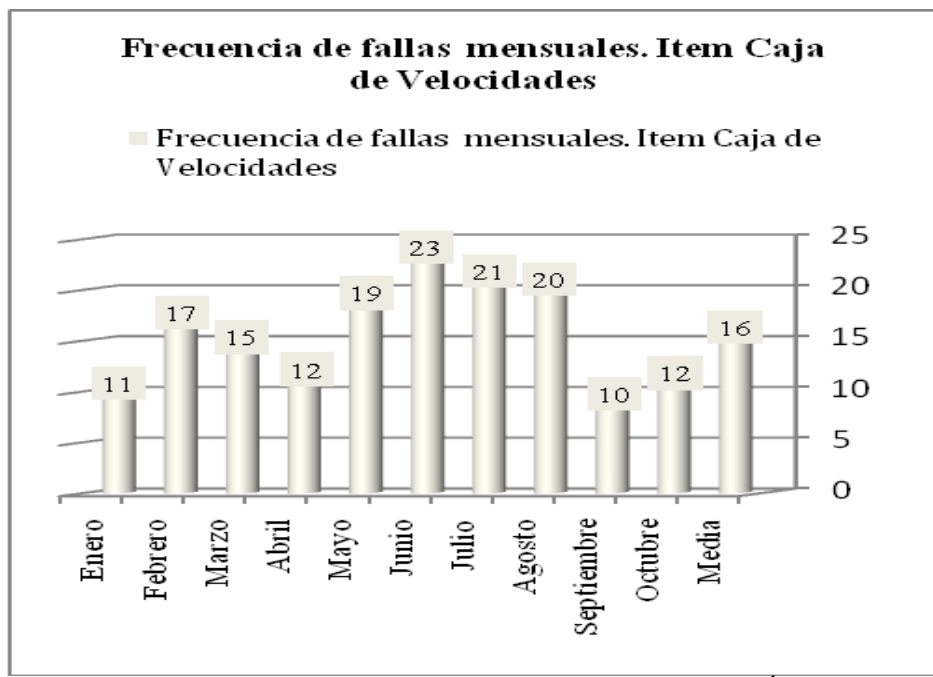

Figura 3 - Frecuencia de fallas en los meses evaluados. Ítem Caja de velocidades.

Fuente: Autores, (2016).

En el caso del ítem puente propulsor la frecuencia siguió una tendencia estable y solo en el mes de julio se observó un incremento de la frecuencia de fallas al fijar un valor de 12 muy por encima de la media que se estableció en 5.5. Las probables causas fueron ya explicadas anteriormente, aunque se destaca el hecho de que existe una tendencia al incremento de fallas de los tres ítems en los meses de julio agosto y setiembre que puede estar relacionado con el incremento también de la temperatura ambiente en este período que a veces llega a valores por encima de $40{ }^{\circ} \mathrm{C}$. Se sugiere realizar un estudio de la incidencia de la temperatura ambiente en la explotación del transporte colectivo.

\section{Frecuencia de fallas mensuales item Puente Propulsor}

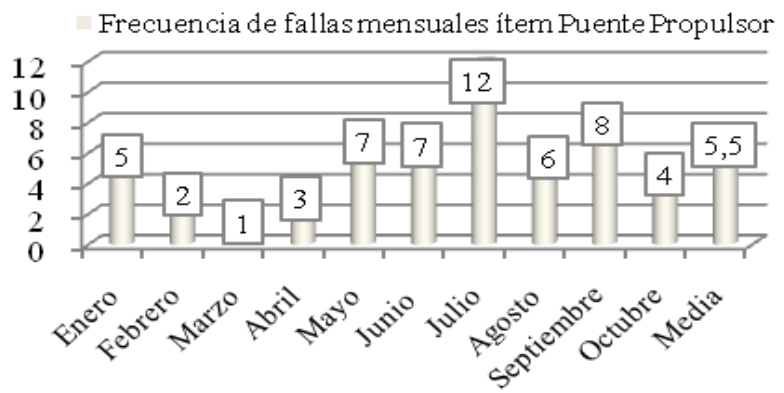

Figura 4 - Frecuencia de fallas en los meses analizados. Ítem Puente Propulsor.

Fuente: Autores, (2016) 


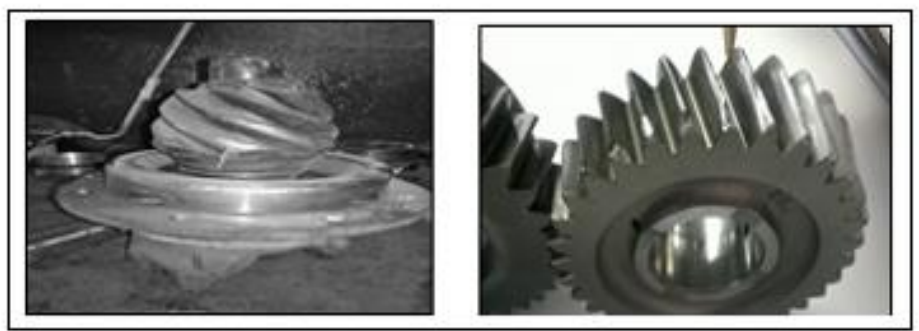

Figura 5: Foto de un engrane de la tercera marcha con desgaste en los dientes y foto de la Corona Principal del diferencial con desgaste.

Fuente: Registros de mantenimiento de la empresa de transporte colectivo urbano, (2014).

Con relación a los principales defectos de estos ítems, las evidencias muestran que existe una frecuencia de fallas o problemas mecánicos mensuales en los tres ítems analizados y que este comportamiento se mantiene en niveles bastante similares de mes en mes cuando en realidad si existiera una buena política de mantenimiento este comportamiento debería ser decreciente.

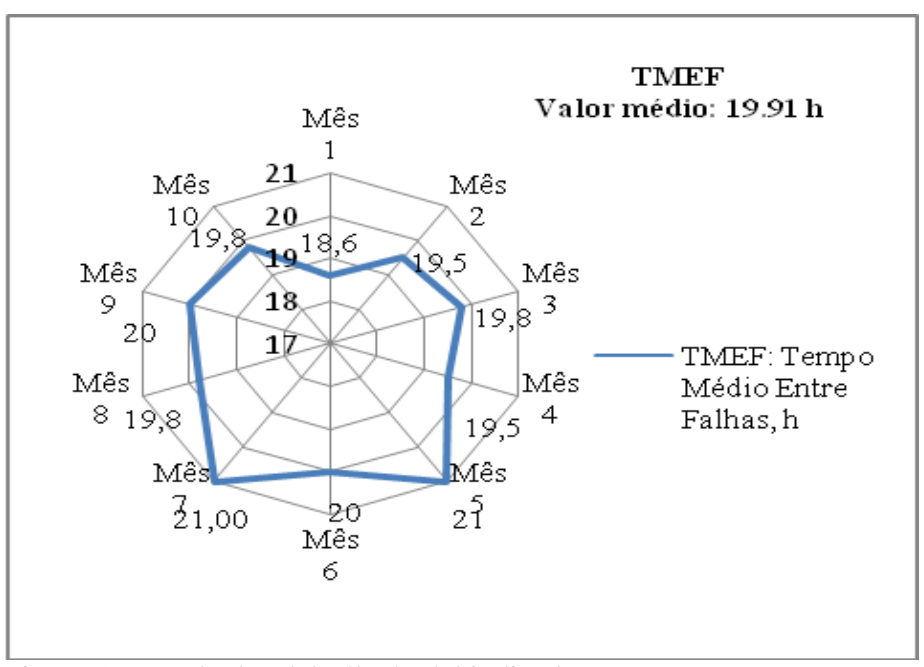

Figura 5 - Resultados del cálculo del indicador TMEF.

Fuente: Autores, (2016).

Analizando los restantes indicadores que corresponden a los llamados índices de clase mundial se tiene que:

Lo valores del indicador tiempo medio entre fallas oscilan en el período analizado entre 21 horas como valor máximo y 18,5 horas como mínimo. Si este valor fuera aumentando con el paso de los meses sería una buena señal porque el mismo indica que el número de intervenciones correctivas iría disminuyendo e consecuentemente el total de horas disponibles para la operación o explotación iría aumentando, en este caso se aprecia una leve mejoría a partir del quinto mes.

Por su parte el resultado del cálculo del indicador TMR muestra que casi no ocurren oscilaciones entre los valores de este indicador por lo que se sugiere consolidar el equipo de mantenimiento y realizar acciones que tiendan a la estabilidad de esta fuerza de trabajo ya que cuanto menor sea este indicador mejor irá el proceso de mantenimiento pues las reparaciones de tipo correctiva aparecerían con menor frecuencia. En este indicador la experiencia, la habilidad y la estabilidad del personal técnico que realiza las labores de mantenimiento tiene una gran incidencia ya que estos pueden llevar a la disminución del mismo.

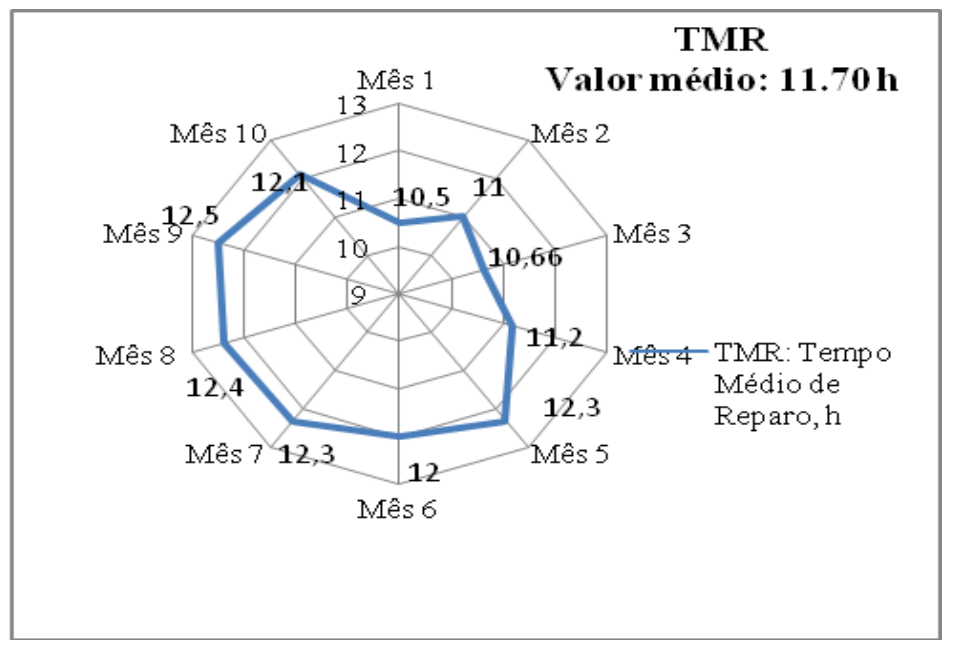

Figura 6 - Resultados del cálculo del indicador TMR.

Fuente: Autores, (2016).

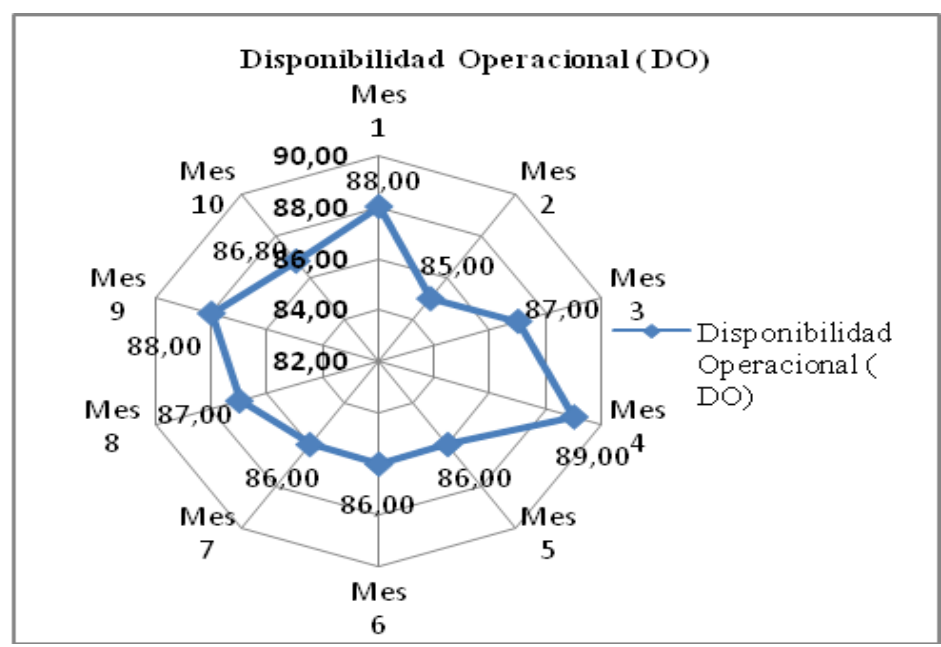

Figura 7 - Resultados del cálculo del indicador DO.

Fuente: Autores, (2016).

El indicador de disponibilidad operativa mostró un comportamiento adecuado con un valor medio de $86.8 \%$ de disponibilidad operativa o lo que es lo mismo existe un $86.8 \%$ del total de las horas disponibles para que estos ítems puedan desempeñar su función adecuadamente, es válido destacar que en este valor no se consideraron las horas de paradas programadas solo las utilizadas para efectuar las labores de reparación tanto correctivas como preventivas que fueron realizadas fuera del tiempo de paradas programadas para mantenimiento.

\section{CONCLUSIONES}

Los resultados del análisis de los indicadores de mantenimiento muestran valores significativos en los tres ítems analizados con una tendencia a incrementos concentrados en los meses de julio a agosto lo que sugiere la incidencia del incremento de la temperatura ambiente propia de la región en esa época del año en la explotación de estos medios técnicos. 
Edry Antonio García Cisneros et al/ ITEGAM-JETIA Vol.02, № 06, pp.55-60. Junho, 2016.

Los resultados del trabajo evidencian la importancia que estos indicadores tienen en las acciones de mantenimiento de las empresas transportistas al facilitar la toma de decisiones con elementos técnicamente fundamentados ya que los mismos permiten evaluar la calidad del mantenimiento, identificar tanto los problemas existentes o como los potencialmente posibles que puedan limitar el rendimiento de estos medios además de que constituyen herramientas de esta actividad que permitirán una mejor distribución de recursos en las labores de mantenimiento.

\section{AGRADECIMIENTOS}

Agradecemos a la Fundación de Amparo a las Investigaciones del Estado de Amazonas (FAPEAM) y al Consejo Nacional de desarrollo científico y Tecnológico de Brasil (CNPq) por el apoyo financiero y técnico para la realización del proyecto de investigación, también a la dirección de la Escuela Superior de Tecnología de la Universidad del Estado de Amazonas (ESTUEA).

\section{BIBLIOGRAFÍA}

[1] ABRAMAN - Associação Brasileira de Manutenção. A situação da manutenção no Brasil. $16^{\circ}$ Congresso Brasileiro de Manutenção. www.abraman.org.br . [Citado 25 Diciembre de 2014.]

[2] Deponti. C. M. Tese de Doutorado em Desenvolvimento Rural. Universidade Federal do Rio Grande do Sul, Faculdade de Ciências Econômicas, Programa de Pós Graduação em Desenvolvimento Rural, Porto Alegre, 2011.

[3] Deponti, C. M.; ECKERT, C.; AZAMBUJA, J.L.B de; Estratégia para construção de indicadores para avaliação da sustentabilidade e monitoramento de sistemas. Agroecologia e Desenvolvimento Rural Sustentado, Porto Alegra, v.3, n.4, out/dez 2002.

[4] Kardec, A.; FLORES, J. F.; SEIXAS, E. Gestão Estratégica e indicadores de desempenho. Rio de Janeiro: Qualitymark, 2002. $120 \mathrm{p}$.

[5] Oliveira A, C. A. de; Rosa, A. D. Motores de combustión interna - alcohol y gasolina. Santa María: CEP SENAI, 2008.

[6] Leandro M. J. Gestão de custos indiretos - custos de manutenção industrial. Revista Eletrônica Lato Sensu, 2008.

[7] PINTO, Alan Kardec e Xavier, Julio de Aquino Nascif. Manutenção - Função Estratégica. Rio de Janeiro: Qualitymark, 2001.

[8] Vianna, H; Ricardo G. PCM Planejamento e controle da manutenção. Río de Janeiro: Qualitymark Editorial, 2008. 